\title{
EDUCACIÓN PARA LA VIDA Y LITERATURA INFANTIL EN LA RED: EL CASO DE DAVIDE CALI
}

\author{
Moises Selfa Sastre \\ Universidad de Lleida \\ Angela Balca \\ Universidad de Èvora (Portugal)
}

\begin{abstract}
RESUMEN: En este artículo presentamos una lectura de tres álbumes ilustrados del escritor de Literatura Infantil Davide Cali que poseen mensajes relacionados con la educación para la vida. Además señalamos la presencia de estos álbumes en la red y las interacciones digitales que los lectores pueden tener con el mensaje literario que Cali propone. Así se destacan diferentes espacios digitales, como blogs, vídeos y podcasts, que facilitan el acceso al texto literario desde diferentes ópticas y perspectivas. La lectura de estos textos, que poseen mensajes subyacentes en cada una de sus páginas, contribuye a la educación para la vida del joven lector, ya que en ellos se presentan conceptos relacionados con esta Educación: el sentido de la vida, el porqué del cumplimiento de las obligaciones cotidianas y las consecuencias del incorrecto ejercicio del poder. A partir de mensajes sencillos y de las ilustraciones que acompañan al contenido textual, Cali y los ilustradores de estos tres álbumes pretenden que el lector tome partido por los ideales allí presentados y se posicione críticamente ante ellos, contribuyendo, de este modo, a su educación para la vida.
\end{abstract}

PALABRAS CLAVE: educación para la vida, literatura digital, lectura, álbum ilustrado, Davide Cali.

\section{EDUCATION FOR LIFE AND CHILDREN'S LITERATURE IN THE WEB: THE CASE OF DAVIDE CALI}

\footnotetext{
ABSTRACT: In this paper we present a reading of three picture books by the writer of Children's Literature Davide Cali that have messages related to education for life. We also note the presence of these picture books on the web and the digital interactions that readers can have with the literary message that Cali proposes. Thus, we stand out different digital spaces, such as blogs,
} 
videos and podcasts, which facilitate access to the literary text from different perspectives. The reading of these texts, which they have underlying messages in each of their pages, contributes to the Education for Life of the young reader, because they present concepts related to this Education: the meaning of life, the reason for compliance of the daily obligations and the consequences of the incorrect exercise of power. Based on simple messages and the illustrations that accompany the textual content, Cali and the illustrators of these three picture books intend that the reader takes advantage of the ideals presented and he is positioned critically before them, contributing, in this way, to their Education for life.

KEYWORDS: Educacion for Life, digital literature reading, picture book, Davide Cali.

Recibido: 25/03/2019

Aceptado: 03/11/2019

Correspondencia: Moisés Selfa Sastre, Universidad de Lleida, Facultat d'Educació, Psicologia i Treball Social, Avinguda de I'Estudi General, 4, 25001 Lleida. Email: mselfa@ didesp.udl.cat.

\section{Literatura INFANTIL en las PRimeras edades: la edUCACIÓN PaRa LA VIDA Y LOS ENTORNOS IMPRESOS Y VIRTUALES DE LECTURA. El CASO DE Davide Cali}

La educación para la vida es un concepto que sitúa el aprendizaje más allá de los muros de la educación formal que se da, principalmente, en las instituciones educativas. Ciertamente, este tipo de educación se ofrece y procura en escuelas, institutos y facultades de formación primaria, secundaria y universitaria, pero no menos cierto es que han surgido nuevas prácticas de educación que sitúa el aprendizaje para la vida en contextos alfabetizadores en los que esta se ofrece con un alto índice de aceptación: bibliotecas municipales, hospitales, parques y diversos espacios de tiempo libre (Lage, 2005: 23).

Ya fue en los años 70 del siglo pasado que Faure, Herrera, Kaddoura, Lopes, Petrovski, Rahnema y Champion (1973) retomaron la crítica de los años 60 a la educación formal de autores destacados como Freire. La educación no debe ser permanente y solo exclusiva para la élite que puede acceder a contextos alfabetizadores. Además, se insiste en la idea de que la lectura es una capacidad que debe trabajarse tanto en contextos de educación formal como en contextos de educación informal.

El aprendizaje para la vida no se organiza a partir de una edad determinada ni en un espacio o tiempo señalado, sino que antes bien la educación para la vida se da en todos los contextos en los que conviven las personas (familia, comunidad, ocio) y supone el trabajo de valores humanísticos ligados al sentido de la responsabilidad y la inclusión social. 
Estamos inmersos en una época en la que conocer y aprender toman un significado por sí mismos. En este sentido, como subrayan Ibarra y Ballester (2010: 9), ya desde las primeras edades hay que enseñar a los alumnos a pensar y a actuar en base a unos criterios y unos principios en los que se sustente la reflexión como motor de la actuación personal. Por tanto, será crucial fomentar el trabajo de las competencias vinculadas a la comprensión y a la comunicación de la información que aparece en textos de diversa índole.

Comprender textos diversos así como la información visual y escrita que aparece en estos es una de las competencias clave en la etapa de la Educación Infantil y Primaria para que el aprendizaje para la vida se dé de un modo funcional y significativo (Martínez Ezquerro, 2016: 10). En este sentido, la lectura y comprensión de textos es un tipo de actividad intelectual compleja, que engloba diversos procesos cognitivos y que, sobre todo, permite la construcción de redes de conocimiento sobre cuyos cimientos se asienta cualquier aprendizaje (Martos y Campos, 2013: 350-415).

A lo largo de las primeras edades, el joven lector posee, desde hace ya hace varias décadas, un tipo y soporte de lectura en los que puede descubrir diferentes mensajes que contribuyan, aunque no exclusivamente, a su educación para la vida. Se trata del álbum ilustrado que Lewis (2001) define como un objeto cultural en forma de libro, fruto de una experimentación entre el lenguaje visual y textual, dirigido tanto a un público infantil como adulto y que es fruto de la evolución cultural, social, tecnológica y artística de nuestro tiempo. Desde este punto de vista, la articulación manifiesta en el álbum entre imagen y palabra es considerada por este autor como una de las formas más extraordinarias e innovadoras de la literatura contemporánea.

La relación entre la ilustración y el texto que ofrece el álbum ilustrado crea en el joven lector una experiencia lectora que lo faculta para otorgar un significado al relato (Ballester, Ibarra y Haba, 2016: 372). Así, el acto de lectura es concebido como la concretización personal por parte del lector de la forma de representación artística propuesta por el emisor. Como afirma Durán (2007: 217), los procesos de recepción lectora y los de percepción visual se equiparan en la medida que generan órbitas cognitivas similares dentro de la mente. Estas órbitas cognitivas gravitan alrededor de los ejes que van del signo al símbolo, de la objetividad a la subjetividad y de la afectividad al ingenio. Estos tres ejes contribuyen a la adquisición de una visión particular, clara y comprensible del mundo y todo lo que rodea a la convivencia personal, lo cual quiere decir que la educación para la vida está asegurada a partir de unos productos de lectura, los álbumes ilustrados, que ofrecen todo tipo de mensajes relacionados con el vivir y relacionarse en el mundo.

La tecnología, paralelamente al libro de texto impreso, ha abierto nuevas posibilidades a la Literatura Infantil (LI) en la medida en que esta puede tener una gran presencia en la red. Consecuentemente, el acceso a esta a través de dispositivos tecnológicos de todo tipo (Colomer \& Fernández, 2014) y en entornos digitales variados provoca que el joven lector pueda consumir LI y conocerla más allá de las fronteras del libro impreso, si bien la lectura en papel no queda para nada excluida, aunque ya no es el único formato a través del cual puede accederse a la LI. 
De este modo, los lectores más jóvenes podrán consumir contenidos de textos literarios propiamente dichos y dominios digitales relacionados directamente con estos. Estos les informarán -en forma de blog digital, por ejemplo- del autor y el ilustrador de la obra, pudiendo interaccionar con ellos en algunos casos. Además les permitirán relacionarse con otros lectores del texto literario consumido en foros digitales, donde se intercambiarán opiniones de todo tipo acerca de la lectura escogida. También existen los booktrailers y los booktubers como canales para promocionar y conversar con otros usuarios sobre las diferentes posibilidades de lectura del texto.

Por tanto, podemos hablar de una nueva LI (Azevedo et al. 2015), asociada a libros y espacios web, y que en sus páginas muestra de forma clara el concepto de dynamic hybrid book acuñado por Dresang (2008) y que Azevedo, Selfa y Balça (2016) relacionan con

Lecturas literarias en entornos digitales e híbridos que permiten al lector acceder a diversas informaciones relacionadas con esta lectura, por ejemplo, los datos del autor, las características de la editorial que publica el texto literario y la interacción con otros lectores del mismo texto a través de códigos y mensajes digitales (p. 107).

Todo ello nos permite referirnos a un espacio híbrido digital, en el que interactúan, en un mismo nivel, diversos agentes de lectura, con un mismo propósito común: el texto literario.

Esta forma creciente de interacción digital ha ido creciendo a lo largo de las tres últimas décadas por tres razones fundamentales que Azevedo, Selfa y Balça (2016) destacaron recientemente:

En primer lugar, la joven generación net ha ido creciendo y construyendo sus conocimientos interactuando con la tecnología digital que la sociedad del conocimiento ha ido poniendo a su disposición; en segundo lugar, las mismas herramientas tecnológicas que los jóvenes lectores manejan han sido usadas por los mediadores de lectura como aliados útiles para fomentar la lectura entre la población juvenil (Martínez Rodrigo y González Fernández 2010); y, en tercer lugar, no puede olvidarse que la inclusión de la tecnología digital en la construcción de cualquier competencia -también la lectora- proporciona una formación completa, integral y sin fisuras en el joven, ya que le capacita para interaccionar con la "sociedad red" (Castells, 2007), concepto referido a los modos de relación e interacción digital entre los ciudadanos alfabetos de un mundo globalizado (p. 107).

Por todo lo aquí expuesto, la educación para la vida no queda excluida de las nuevas formas de interacción digital que hoy en día ofrece la LI, sino que esta educación puede darse igualmente a través del consumo de textos literarios infantiles en la red, en el que pueden discutirse y replantearse los mensajes que el autor quiere ofrecer al autor para que se posicione en un sentido u otro.

Davide Cali es uno de los más actuales creadores de álbumes ilustrados. Nació en Suiza, si bien creció en Italia. Como autor de álbumes ilustrados, ha creado más de 
40 obras en 10 años, publicadas en Francia (Sarbacane, Actes Sud, Thierry Magnier, Gulf Stream, Trimestre, Rue du Monde, Nobi-Nobi), Austria (Annette Betz), Portugal (Planeta Tangerina, Bruua), Italia (Zoolibri, Arka, Kite), España (Edelvives, Zorro Rojo, Faktoria $\mathrm{K}$ de libros, SM) y más de veinte países en los cinco continentes. También ha publicado bajo el pseudónimo de Taro Miyazawa en la editorial francesa Michel Lagarde. Sus obras han recibido varios premios en Francia, Bélgica, España, Suiza y Alemania. Varias compañías de teatro han Ilevado al escenario dos de sus obras más famosas, como por ejemplo Moi, j'attends (2005) [El hilo de la vida]. Sus cómics para niños aparecen publicados en las revistas mensuales Mes Premiers J'aime Lire, y en los álbumes publicados por Sarbacane, mientras sus cómics para adultos aparecen en L'Echo des Savanes y en Fluide Glacial.

La presencia de la figura de Davide Cali en la web podemos descubrirla a partir de la interacción que este ofrece con el público lector en su página digital oficial: www. davidecali.com. Esta página, en constante remodelación y actualización, permite conocer la obra escrita de este autor infantil en lengua original, así como traducciones a otras lenguas. También permite interaccionar con el autor a partir del acceso a su Facebook (https://es-es.facebook.com/Davide-Cali-164285603678359/) y a otros entornos de relación como, por ejemplo, el entorno Messenger. El lector puede estar acceder a las novedades editoriales de Cali y la difusión de estas en espacios de educación formal y no formal. Véase, por ejemplo, el enlace https://www.bm-reims. $\mathrm{fr} /$ Default/doc/SYRACUSE/841500 que informa de la última publicación de este autor franco-suizo: Petit Pois (2017).

Davide Cali presenta en sus álbumes ilustrados mensajes subliminales que comprometen al lector aunque este no quiera. Se trata de mensajes que están relacionados, en muchas ocasiones, con el quid de la vida y los derechos y deberes de la condición humana. Por tanto, son propuestas que rápidamente conectan con el lector, joven aunque también adulto, y que le obligan a posicionarse ante el dilema que el texto ilustrado plantea. Desde este punto de vista, los objetivos de este estudio son tres: a) mostrar la presencia en la red de los álbumes ilustrados que de Davide Cali hemos escogido; b) destacar y analizar los conflictos que aparecen en esos tres álbumes: el sentido de la vida, el porqué de las obligaciones diarias y el ejercicio del poder; y c) analizar qué suponen estos tres conflictos en relación con una educación para la vida. Para ello, realizaremos una lectura hermenéutica de tres álbumes ilustrados de Davide Cali que tratan sin tapujos realidades ligadas a la existencia humana. Esta lectura crítica y reflexiva de estas cuatro obras nos permitirá destacar la emergencia de temas y valores relacionados con la interpretación del texto, presentes en niveles de lectura menos evidentes y más profundos.

\section{El SENTIDO de la VIDA: MoI, J'AtTends (2005) [EL hILO DE LA VIDA (2017)]}

El primer álbum al que nos vamos a referir es Moi, j'attends (2005), Premio Baobab en Montreuil (2005). Este texto fue escrito originalmente en francés y publicado por Sarbacane. La traducción al inglés Ileva por título I can't wait (2005) y al español el de El hilo de la vida (2017). Las ilustraciones son de Serge Bloch, ilustrador de 
libros infantiles y juvenil además de dibujante en periódicos como The Washington Post y The Wall Street Journal.

El hilo de la vida es un álbum de tapa dura en el que destaca la supremacía de la horizontalidad de la maquetación sobre la verticalidad de esta. Esto es así porque este texto utiliza la metáfora de un hilo rojo, largo y dispuesto en una línea horizontal para enmarcar y destacar las diferentes etapas de la vida de una persona cualquiera, desde que nace hasta que muere. En efecto, este álbum nos presenta a un personaje, el cual, cogido de un hilo rojo, va superando las distintas etapas vitales de un ser humano común.

Un paratexto textual que es necesario destacar es el de la contraportada del texto. En la parte superior central encontramos la primera clave para la correcta interpretación del texto: "Suivez le fil de la vie... Un livre pour tous les âges. Un cadeau pour toujours" (Cali y Bloch, 2005, s/p). Junto a este texto, encontramos la imagen de un niño que, en posición agachada, quiere tomar con la mano un hilo rojo que lo conduce a la portada del álbum, en la cual aparece este mismo niño convertido en todo un hombre que ha encontrado el amor junto a la mujer. El símbolo de este amor es un corazón dibujado con el hilo rojo que acompaña al protagonista a lo largo de todo el texto. Por tanto, el lector, infantil, juvenil o adulto, entiende que la vida está compuesta de ciclos vitales y que cada ciclo lleva consigo un conjunto de vivencias que forman parte de la educación y formación de la persona.

El hilo de la vida es un texto literario que el lector más joven puede gustar en entornos digitales diversos en formato de vídeo para IPhone / IPad (https://www.idboox. com/applis-et-ebooks-enfants/moi-jattends-un-petit-bijou-numerique-tire-du-livrepapier/) y Android a través de la aplicación de Youtube: https://www.youtube.com/ watch? $\mathrm{v}=\mathrm{i} 2 \mathrm{Ymk} 0 \mathrm{VI} 7 \mathrm{~ms}$ En ambos entornos se presenta la historia lineal del texto, si bien en la versión para IPhone / IPad el lector puede construir su propia historia . Esta construcción personal no desvirtúa el propósito de Cali de ofrecer un texto de tipo existencial, metáfora en toda regla del sentido de la existencia humana y de las fases de la vida. También existen blogs que informan de la sinopsis de este texto, de sus características físicas y señas de identidad (https://www.megustaleer.com/libros/el-hilode-la-vida/MES-097906) así como la explicación mitológica del hilo rojo de la vida, que es el motivo sobre el cual Davide Cali construye su texto para los más jóvenes: https://blog.caminosconsciencia.es/el-hilo-rojo-de-la-vida-davide-cali-y-serge-boch/. Este blog contiene un podcast de casi 23' que narra la leyenda de este hilo y su origen en relación a la existencia humana. Los dos blogs citados facilitan la interacción, a través de entradas de comentarios, entre los usuarios digitales de este texto literario.

El hilo de la vida es un texto compuesto de cuatro partes que coinciden con las cuatro etapas de la vida: la niñez, la adolescencia y juventud, la madurez y la vejez. Estas etapas son fácilmente reconocidas porque cada una de ellas empieza con un J'espère [Yo espero]. Las cuatro etapas constituirán una ocasión para la educación

1. Las citas textuales de los tres álbumes ilustrados a los que nos referiremos en este estudio, con la finalidad de respetar su formulación inicial, las presentamos en la lengua original en las que estos fueron escritos, no en las traducciones de estas tres obras al español. 
para la vida, ya que el protagonista tendrá que afrontar los acontecimientos que le son propios en cada uno de los ciclos vitales.

En la primera de ellas, la de la niñez, el protagonista espera que sucedan cinco situaciones propias de este ciclo vital: hacerse mayor, un beso de sus padres antes de ir a dormir, que esté listo el pastel que ha preparado mamá, que deje de llover para salir a la calle y que Ilegue la Navidad. Las ilustraciones presentan esta actitud de espera que, en todos los casos, supone una aceptación de que los acontecimientos vitales no siempre llegan cuando una persona quiere que lleguen, sino que en muchas ocasiones hay que saber esperar para que las cosas se den a su tiempo justo. Así, cuando el protagonista espera hacerse mayor, aparece tirando con fuerza y con resignación un hilo rojo que quiere simbolizar las ansias del niño por hacerse mayor. Como sabemos, esto no es así de rápido y de automático. Por tanto, el lector aprende a esperar y a ir pasando por cada uno de los momentos que hay que transitar desde la niñez a la adolescencia. Lo mismo puede decirse cuando el niño protagonista espera que deje de llover para salir a la calle o que Ilegue la Navidad. Cuando esta ha Ilegado, su padre decora el árbol de Navidad con el hilo rojo que acompaña al personaje desde el inicio del álbum.

La segunda etapa que aparece representada en la vida es la de la adolescencia y juventud. Esta es representada por Cali y Bloch con un joven que, bien cogido del hilo rojo de la vida, espera el amor: "J'attends I'amour" (Cali y Bloch, 2005, s/p). Una vez encontrado el amor, el protagonista espera volver a ver a la chica que le gusta, a recibir una carta de esta y a que "elle me donne oui" (Cali y Bloch, 2005, s/p). Una vez conseguido este sí, el joven tira de un hilo rojo y horizontal en el que en el otro extremo se ve a su prometida vestida de novia camino del altar. Por tanto, podemos decir que se ha consumado una segunda etapa que ha significado la consolidación pública de un amor y el inicio de una relación en una nueva familia a punto de constituirse. A diferencia de la primera etapa de la niñez, aquí el joven protagonista no observa con resignación cómo la vida tiene su propio devenir y que los acontecimientos deben sucederse a su tiempo, sino que en esta etapa de la juventud el personaje muestra cómo con su trabajo y tesón es capaz de alcanzar el amor anhelado. Junto al amor, aparece en esta segunda etapa otra de las preocupaciones de la juventud: el fin de la guerra. Aunque Cali y Bloch solo se refieren a ella en una única lámina del álbum, es necesario referirse a ella: el joven siente cómo la vida de un herido de guerra, que puede ser él mismo, corre peligro a no ser por el hilo rojo que conecta su cuerpo con un gotero. De ahí que Cali y Bloch quieran educar al joven lector en la necesidad de evitar las confrontaciones para un correcto crecimiento en la vida, además de mostrar la obviedad y el significado de que la vida humana pende de un hilo.

La tercera etapa es la de la madurez. El protagonista, una vez encontrado el amor, avanza en la vida. El siguiente estadio es el de esperar un bebé. El amor entre un hombre y una mujer da frutos y lleno de júbilo nuestro personaje exclama "J'espère... un bébé" (Cali y Bloch, 2005, s/p). Esta espera queda simbolizada con el jersey que con hilo rojo teje la futura madre para su bebé. Y no todo va a quedar reducido a la espera un bebé, sino que se espera algo más: "savoir si ce sera un garçon ou une fille" (Cali y Bloch, 2005: s/p). El bebé sale del vientre de su madre acompañado de un 
hilo rojo que simboliza la ligazón para toda la vida del protagonista con el hijo que acaba de nacer. La responsabilidad de los padres hacia sus hijos es manifestada en las siguientes láminas del álbum: el protagonista espera que sus hijos crezcan y que pronto lleguen las vacaciones para pasarlas junto a ellos.

Otro de los aprendizajes de la vida que aparecen en esta tercera parte es el de aprender a perdonar y a pedir perdón en las relaciones de pareja: "... laisser l'autre demander pardon" (Cali y Bloch, 2005: s/p). La ilustración de este aprendizaje no puede ser más explícita: un hombre y una mujer distantes, y un hilo rojo y enredado que parece separar el corazón de ambos. Cali y Bloch están motivando al lector, no olvidemos que niño pero también joven o adulto, a superar las diferencias a partir del diálogo sereno entre dos personas.

La cuarta parte del álbum ilustrado está dedicada a la vejez y a las situaciones vitales ligadas a ella. El protagonista, junto a su esposa, espera, con cierta ansiedad, a que sus hijos, ya mayores, lo llamen por teléfono. En este contexto vital observamos la simbología del hilo rojo que es el canal telefónico de comunicación entre padres e hijos. Junto a estas situaciones de espera, aparecen las enfermedades. Ahora, el protagonista espera a que "le docteur dit: Ce ne sera rien" mientras ausculta a su mujer. Parece que esto no será así, ya que en la siguiente lámina se dibuja ya la muerte de la esposa, que inevitablemente muere, con lo que este hilo de la vida que lo liga a la difunta queda roto. En la ilustración se muestra el cortejo fúnebre presidido por el viudo que obsequia a su esposa una corona de flores tejida con el hilo rojo de la vida. El álbum finaliza con lo último que espera el personaje: que sus hijos vengan a visitarlo y que de ellos nazca nueva vida: "que bientôt il y aura une nouvelle vie dans la famille" (Cali y Bloch, 2015). Se cierra de este modo el ciclo vital del protagonista que arranca en la niñez y finaliza cuando este, ya en la senectud, espera con ilusión el nacimiento de sus nietos. En la última lámina del libro encontramos el hilo rojo perfectamente recogido en un ovillo que simboliza que todo en la vida ha estado ya hecho y que cada acontecimiento vital ha sido una ocasión de aprendizaje.

El hilo de la vida es un álbum ilustrado que invita al lector a tomar consciencia del ciclo vital y del aprendizaje para la vida. En cada una de las etapas de la vida se presentan un conjunto de situaciones que son motivo de reflexión para el lector. Este, tomando partido en cada una de estas, aprende a situarse frente a la vida y a actuar en ella de un modo muy determinado y particular. No se trata solo de reflexionar sobre lo que le sucede al personaje protagonista, sino sobre todo de aprender y tomar partido, en una dirección u otra, con la cual sentirse identificado.

\section{EL PORQUÉ DE LAS OBLIGACIONES DIARIAS: I DIDN'T WORK MY HOMEWORKS BECAUSE ... (2014)}

El segundo álbum que vamos a analizar está escrito originalmente es inglés y tiene como título I didn't do my homeworks because ... Fue publicado en 2014 por Chronicle Books LLC e ilustrado por Benjamin Chaud, prolífico dibujante francés que en el año 2011 recibió, de parte del Gremio de Libreros de Madrid, el premio al mejor álbum ilustrado por su trabajo de ilustración en Pomelo crece (2011). La 
traducción de este álbum al español lleva por título el de No he hecho los deberes porque... (2014).

El título del álbum es muy sugerente ya que, sin duda, estimula la imaginación del lector que tendrá que descubrir por qué el protagonista del texto no ha hecho los deberes escolares, una tarea que está íntimamente ligada al quehacer cotidiano de los jóvenes estudiantes. Este trabajo de realizar las tareas escolares en casa forma parte de la educación en la vida de la población infantil y juvenil.

El primer paratexto que queremos destacar es el color verde intenso de la portada y contraportada. Es un color que pertenece a un reptil que sobre su boca sostiene al joven protagonista, que parece querer alcanzar su mesa de escritorio en la que están sus hojas de tareas escolares. Por tanto es este reptil el que seguramente no deja cumplir con sus obligaciones escolares al protagonista, que se debate entre la realidad de tener que hacer los deberes o divertirse con este animal que ha invadido su vida.

En la contraportada encontramos un segundo paratexto a destacar. Los autores de este álbum ilustrado advierten al lector cuándo debe usarse este libro: "As a long haven't done your homeworkss" (Cali y Chaud, 2014: s/p). Y junto a esta advertencia una segunda recomendación: "Attetion: You use each excuse only once" (Cali y Chaud, 2014: s/p). Por tanto, no existe una recomendación explícita de los autores de este álbum a no cumplir con las tareas escolares, pero si alguna vez el lector no las cumple, puede echar mano de este texto para justificar una actitud que, en principio, no es justificable.

No he hecho los deberes porque ... es un texto al que puede aproximarse en la red el lector a través de booktrailers, como los de https://www.youtube.com/ watch?v=VCU4lcAv5Oo y https://www.youtube.com/watch?v=mCy_CMWrWug, o booktubers, como en https://www.youtube.com/watch?v=u1DI9k5GuDY. En el segundo booktrailer citado, se presenta la historia a dos voces: la de un adulto, que es la profesora que interpela al niño estudiante de por qué no ha hecho sus tareas escolares, y la voz de este niño. Por tanto, a través de un video alojado en el entorno de Youtube, el lector interactúa con un texto que es presentado a modo de diálogo como si de una lectura dramatizada se tratase. En el caso del booktuber se destacan las claves de lectura del texto. Así, emerge el valor de la imaginación para justificar el incumplimiento de las obligaciones personales. Además, no todo es justificable: las mentiras y las excusas no son justificantes suficientes para no asumir con responsabilidad las tareas escolares.

Como en el caso del primer texto comentado en este trabajo, No he hecho los deberes porque ... también posee blogs para que interaccionen los lectores acerca de lo que les sugiere esta lectura. Es el caso del blog http://entremontonesdelibros. blogspot.com/2014/12/deberes-cali-chaud-mientrasleo.html, en el que podemos leer cuarenta y siete comentarios que tanto jóvenes lectores como también adultos mediadores de lectura plasman en la red sobre lo que les ha sugerido la lectura del texto de Cali. Estos comentarios, en su mayoría, pretenden justificar al protagonista de este álbum y las razones que este aduce para no haber cumplido con las tareas escolares. 
El álbum comienza con una interrogación directa de la profesora, que esconde detrás de su espalda y cogido de sus manos un pequeño libro de color verde, al joven lector: "So... tell me, why haven't you do your homeworks?" (Cali y Chaud, 2014: $\mathrm{s} / \mathrm{p})$, a lo que el protagonista responde no sin poner una cara de extrema sorpresa: "I didn't do my homeworks because..." (Cali y Chaud, 2014: s/p). Y es aquí donde empiezan las veintiocho excusas que con las que el protagonista intentará convencer a su incrédula profesora.

Estas veintiocho razones de ninguna manera justifican esta actitud del protagonista de no hacer sus deberes, pues todas ellas están relacionadas con sucesos irreales y que forman parte del mundo imaginario del joven. Son veintiocho excusas que pueden ser agrupadas en cuatro bloques. El primero de ellos tiene que ver con los animales que impiden al protagonista realizar sus tareas escolares. Entre ellos, podemos destacar los siguientes: un avión de monos que aterrizó en el jardín de su casa; unos enormes reptiles que invadieron su barrio; un perro que se traga al perro del protagonista, con lo que este debe acudir al veterinario para solucionar esta cuestión; la muerte del gato del educando que acude triste a su entierro; unos enormes pájaros de plumas rojas y negras que construyeron un enorme nido en el tejado de la casa del protagonista y un pingüino perdido que tuvo que ser llevado al Polo Norte por este joven niño. Cuando este enuncia esta excusa, la maestra lo interrumpe y le indica que los pingüinos no viven en el Polo Norte, a lo que el protagonista responde, no sin advertir su error fruto de la inseguridad y por qué no de sus falsedades conscientes, que "effectiely! When we realized our mistake we had to turn around and take it to the other Pole..." (Cali y Chaud, 2014: s/p). Esta advertencia de la maestra es, pues, para el lector de este álbum una invitación a decir la verdad aunque no hayas cumplido con tus obligaciones.

Un segundo bloque de excusas tiene que ver con la aparición de seres y objetos fantásticos que impiden al niño estudiante realizar sus tareas escolares. Destacamos, sobre todo, tres de ellas por la irrealidad que las caracteriza y que el lector percibe como tal, no sin provocar en él una sonrisa cómplice acentuada por las ilustraciones que acompañan al texto: un robot descontrolado y gigante destrozó su casa; fue abducido por un OVNI; fabricó con su tío una enorme y compleja máquina de color negro para realizar los deberes, pero esta no llegó nunca a funcionar.

El tercer bloque de excusas está relacionado con personajes del imaginario infantil que el lector reconoce como tal ya que forman parte de otros textos infantiles y juveniles de distintas épocas y géneros: se trata de duendes que esconderán los lápices con los que el joven estudiante debe realizar sus tareas escolares, de vikingos que con sus galeras atacaron la habitación de este, de presos fugados de la cárcel que se escondieron en la habitación del protagonista y que este no logró echar, de Robin Hood a quien el protagonista le regala sus lápices y de un famoso director de cine que invadió el escritorio del protagonista para rodar su nueva película.

Por último, el cuarto bloque está relacionado con un conjunto de acciones cotidianas que impedirán la realización de las tareas escolares y que todas ellas afectarán al protagonista y a su familia. Son acciones que pueden ser creíbles, pero que el lector percibe como no justificables en relación a las obligaciones del estudiante que debe 
hacer sus tareas escolares: su familia se queda sin leña, con lo que tuvo que sacrificar sus cuadernos escolares para calentar a su padre, madre, hermana y perro; su familia encontró petróleo en su jardín que manchó sus cuadernos escolares, su familia tuvo que ayudar a sus vecinos a encontrar sus armadillos y, finalmente, la mascota de su hermana devoró los lápices y cuadernos del protagonista con lo que no pudo realizar sus tareas escolares.

Después de enunciar estas veintiocho excusas, el protagonista, con cara de ingenuo, interpela a su maestra así: "That's why haven't been able to do my homeworks..." (Cali y Chaud, 2014: s/p), si bien la maestra parece no creerlo como el lector percibe a través de la ilustración de esta que agacha su espalda y con sus ojos fija la mirada en el rostro del niño. Ante tal situación el protagonista dice a su maestra: "Why doesn't he believe me?", La maestra le responde con una sonrisa pícara: "Because I have read the same book" (Cali y Chaud, 2014: s/p). Y le enseña el libro que escondía detrás de su espalda que no es otro que el de Cali y Chaud (2014) I didn't do my homeworks because...

Con esta respuesta la maestra quiere educar al niño en la vida en dos aspectos fundamentales: a) la necesidad de cumplir con las obligaciones escolares diarias que contribuyen a la educación del niño y b) la importancia de decir siempre la verdad a pesar de no haber cumplido esas obligaciones. El álbum finaliza con las copias que la maestra encomienda al protagonista. En estas copias aparece la siguiente oración: "Any giant reptile invaded my neighborhood" (Cali y Chaud, 2014: s/p).

En este álbum ilustrado, Cali y Chaud (2014) nos presentan un tema que forma parte de la educación en la vida de los jóvenes lectores: la importancia de cumplir con sus tareas escolares a pesar de ser esta una tarea que pueda despertar desidia en esta población. Se trata de educarlos en la responsabilidad que, con el paso de la vida, irá siendo cada vez mayor en relación a obligaciones de mayor envergadura y trascendencia, que a buen seguro que irán más allá de la realización de los deberes escolares.

\section{El correcto ejercicio del poder para la buena convivencia: $\boldsymbol{A}$ RAINHA DAS RAS NAO PODE MOLHAR OS PÉS (2012)}

Este tercer álbum ilustrado fue traducido y publicado en portugués por la editorial Bruaá Ediçao e Design en el año 2012. El texto inicial fue entregado a esta editorial por Davide Cali en francés y fue Miguel Gouveia, de la editorial portuguesa antes indicada, quien lo tradujo a la lengua portuguesa. Las ilustraciones de la edición en portugués corren a cargo de Marco Somá, ilustrador de libros infantiles cuya obra ha sido seleccionada para ser expuesta en la Feria del Libro de Bolonia (2011, 2013, 2014 y 2016). Además, cuenta con numerosos premios, como el XIII Premio Emanuele Luzzati por las ilustraciones en La regina delle rane non può bagnarsi i piedi (2013) y el XV Premio Giovanni Arpino por Robot (2014). Una información detallada en la web de este ilustrador puede consultarse en http://marcosoma.blogspot.com/. En esta, el ilustrador ofrece al joven lector la posibilidad de interaccionar con él a través de diferentes portfolios clasificados en edades: 0-7 años y 7-11 años. Posteriormente 
A rainha das ras nao pode molhar fue traducido a otras lenguas, como por ejemplo el español: La reina de las ranas no puede mojarse los pies (2013) por la editorial Libros del Zorro Rojo.

A rainha das ras nao pode molhar os pés plantea al joven lector una lección de vida sobre el correcto ejercicio del poder y la autoridad. Del correcto ejercicio del gobierno y de la dirección que se le quiera dar a este dependerá la felicidad y el bienestar de las personas y de quien ejerce la potestad. Este correcto ejercicio es el que se quiere destacar en la red a través de booktrailers diversos sobre esta obra. Destacamos dos de estos booktrailers: (https://www.sinembargo.mx/27-02-2016/1628900 y https://josehuedo.wordpress.com/la-reina-de-las-ranas/). En este último, a través del entorno digital Vimeo, se presenta la historia con todos los conflictos que se derivan de esta. También el lector infantil puede visualizar este texto literario a partir de su representación por cuentacuentos profesionales: https://www.youtube.com/ watch? $v=$ YryHlb2WMMY. Pero de todos los entornos digitales en los que tiene presencia esta obra, queremos destacar el de https://darabuc.wordpress.com/tag/davidecali/. Darabuc, pseudónimo literario de Gonzalo García Rodríguez, ofrece su visión particular de esta obra desde una perspectica de adulto experto en LI:

El texto reelabora el cuento en una variante que se aleja de algunas posibilidades del original, como son lo brusco y lo grotesco. También se aleja (sobre esto valdría la pena pensar, creo, y más en un libro de origen portugués) de las posibilidades políticas de la historia, que hoy son muchas y muy idóneas. Pero esto no lo pretenden los autores y entiendo que la línea elegida la desarrollan con riqueza y acierto, y en cuanto a posibles usos para la reflexión moral (implícita en tanta fábula), diría que es un buen álbum para determinados conflictos de patio. (Darabuc, 2012).

Por tanto, se interacciona con el potencial lector a partir de la enseñanza moral que este texto de LI quiere ofrecer. Esta entrada de blog es comentada por dos lectores que ofrecen su visión particular de este álbum ilustrado. Del mismo modo, existe en español otro blog (https://biblioabrazo.wordpress.com/2013/09/16/la-reinade-las-ranas-davide-cali-y-marco-soma/) en el que el texto de Cali es ampliamente comentado por numerosos lectores. Estos destacan la importancia del tema que Cali y Somá quieren potenciar con su obra: el poder no da derecho a ejercerlo con absoluta impunidad y sin pensar en el bien común.

La obra de Cali y Somá (2012) es una historia que empieza en un día cualquiera en el que aparentemente no sucede nada extraordinario. Los personajes de esta son unas ranas, caracterizadas como humanas a través de las acciones que realizan y de unas deliciosas ilustraciones en tonos marrones y verdes claros, que viven en una charca en un clima de fraternidad: "Havia uma vez um lago e na lagoa havia sapos. Os sapos passariam o dia fazendo coisas de rãs: eles iriam pular e caçar voar, sonhar ou brincar com libélula" (Cali y Somá, 2016: s/p). Hasta aquí todo transcurre en una aparente normalidad, que se ve alterada por un objeto que, en una noche de verano y después de cenar, entra en escena: una corona que caída del cielo irrumpe en la vida de los anfibios. Una de estas ranas observa dónde cae exactamente esta corona y se lanza a por ella. Con sus manos la alcanza y se la coloca en su cabeza sin saber 
qué hacer. El resto de las ranas la aclaman y se precian de tener una compañera como reina: "Olhe! É a rainha das rãs! [...] -Hurra para a nossa rainha! [...] Tudo imitado e a rainha das rãs foi aclamado entre grande aplauso" (Cali y Somà, 2012: s/p). Es decir, Cali y Somá (2012) quieren darle a este acto de proclamación de una rana reina una aparente normalidad, ya que se trata de una igual a las otras ranas y que va a ejercer su soberanía sin alterar la normal convivencia entre los animales del estanque.

No obstante, eso no fue así. A partir de ese día la reina rana tenía consejeras, criadas que le cazaban moscas para comer y otras que hacían exhibiciones para divertirla: "Uma rainha não pode falar com as outras rãs, não pode molhar os pés e deve ter um grande olho nenugar para si mesma. Uma rainha de rãs não pode ficar cansada, deve dormir muito, comer apenas moscas gordas, dar ordens e punir rãs se eles não obedecerem imediatamente" (Cali y Somà, 2012: s/p). Pero pronto alguien en el estanque empezó a cuestionar su derecho a tener tales privilegios por el simple hecho de haber encontrado una corona y habérsela colado en su cabeza: "-Mas, por que una rã que encontra uma coroa se tornou uma rainha de rãs? -insistiu a rã (Cali y Somá, 2012: s/p).

Los días en el estanque pasaban y cada día la reina de las ranas exigía más y más privilegios. Un día, las ranas organizaron un concurso de saltos. Saltaron todas las ranas y el salto final debía darlo la reina, a la que le habían reservado el privilegio de dar el último y más espectacular salto. La reina rana no quería saltar, pero no tuvo más remedio que hacerlo como reina que era: "Por que a rainha tem que pular?", Perguntou una rã conselheira. -Porque uma rainha é sempre a melhor que faz tudo, e, portanto, estamos esperando ver seu salto maravilhoso." (Cali y Somá, 2017). Tomó impulso y bajó hasta el fondo. Al salir del agua, la corona que llevaba en su cabeza desapareció y la reina rana ordenó buscarla, a lo que el resto de las ranas se negaron porque ya no llevaba en su cabeza la corona y no podía, en consecuencia, ordenarles nada:

"Procure minha coroa!"-ele ordenou.

-Por que nos está dando ordens? -perguntaram as rãs.

-Porque eu sou sua rainha!

-Se não usar uma coroa, não pode ser uma rainha! (Cali y Somá, 2017: s/p).

De pronto empezaron a tirarle bolas de barro hasta que un hombre, a bordo de una barca, alcanzó la corona de la reina de las ranas y se la llevó consigo. La desaparición del estanque de tal objeto fue el motivo para que la normalidad regresase a la vida de todas las ranas y a que ya no hubiese más reinas en el estanque: "Não há mais rainhas e cada rã caçasuas próprias moscas." (Cali y Somá, 2017: s/p).

Este álbum ilustrado presenta una temática muy útil para reflexionar con los jóvenes lectores en relación con la Educación para la Vida: el incorrecto ejercicio del poder provoca en los demás situaciones de rechazo hacia quienes lo ejercen de forma despótica. En este sentido, la lectura de este texto invita al lector a pensar sobre las obligaciones que conlleva ser reina, además de cuestionar el mal uso del poder para la feliz convivencia del grupo. El poder hay que ejercerlo con responsabilidad 
y decoro y, como quieren mostrar Cali y Somá (2017) al joven lector, el ejercicio de este está reservado para sujetos capaces de ocuparse del bien común y no del propio. Si no se hace así, la desigualdad entre iguales es evidente, con lo que el inicio de conflictos en la comunidad está asegurado.

\section{Conclusiones}

El objetivo de cualquier iniciativa de tipo educativo lo constituye, en el fondo, la formación para la vida. La educación ya no debe basarse en un programa preestablecido que hay que explicar desde un punto de vista meramente teórico, sino, como sostiene Guarisma (2007: 1-2) en el conocimiento práctico de la vida humana. A la luz de esta convicción, la educación del niño adquiere, sin duda, gran importancia.

Como seres humanos que somos, seres en definitiva sociales, nos movemos a través de paradigmas. Actuamos en el mundo según esos modelos, que podríamos equiparar con mapas preestablecidos. Si bien los paradigmas de cada sujeto son respetables, no podemos decir que sean una cosa únicamente subjetiva: hay modelos que funcionan y otros que no.

Los modelos de conducta pueden ser apreciados y aprendidos por el niño y el joven a través del consumo de diferentes productos culturales: Internet, cine, televisión y, como se ha visto en este artículo, la lectura de álbumes ilustrados y entornos digitales múltiples y variados. En este sentido, la extraordinaria revolución que han consensuado las herramientas digitales para interaccionar a través de la banda ancha en Internet (4G) posibilita el acceso de los jóvenes lectores a nuevas formas de lectura en la pantalla y a interaccionar con los autores y otros lectores de las obras consumidas.

De este modo, existe una "lectura polifónica y marcadamente abierta" (Azevedo, Selfa y Balça, 2016: 116), en el que diferentes agentes relacionados con la lectura interaccionan en un mismo nivel, en un espacio abierto y dispuesto para el diálogo entre iguales, que siempre tiene como eje vertebrador el texto literario y las posibilidades interpretativas que este ofrece.

Los álbumes ilustrados de Davide Cali aquí analizados presentan al lector tres aspectos fundamentales para la educación para la vida: qué sentido tienen la vida y las diferentes etapas de estas, las obligaciones diarias como una oportunidad de formación personal y el correcto (o no) ejercicio del poder cuando este se posee. Son tres aspectos que obligan al lector a posicionarse a partir de la lectura de los álbumes ilustrados. El mensaje de estos para nada lo dejan indiferente, ya que como ser social no puede rehuir lo que los autores de estos quieren transmitir. No se trata tanto de estar de acuerdo o no con el mensaje que subyace en cada álbum. Antes bien, de lo que se trata es de adoptar una postura crítica a través de la lectura de un texto que ha sido especialmente creado para el joven lector $y$, en consecuencia, para la educación para la vida de este.

En el primer álbum destacado en este artículo, Cali y Bloch destacan el papel cíclico de la vida en un intento que va más allá de presentar los ciclos vitales. Lo que en el fondo se pretende es destacar la importancia de cada momento de la vida sin 
añorar el pasado y desear ardientemente el futuro. En el segundo álbum analizado presenta la necesidad de cumplir con las obligaciones cotidianas aunque no sean del agrado del sujeto. En las edades iniciales de la vida, esas obligaciones humanas están ligadas, en general, a las tareas escolares, pero estas no son más que la preparación para cumplir en el futuro con obligaciones profesionales y sociales que pueden no ser del agrado de la persona. En el tercer álbum, se dibuja el mensaje de que el ejercicio del poder tiene que conducir a pensar en el bien colectivo y no en el personal. La figura de la rana no es más que ese lector inicial que en el futuro puede ostentar un cargo de responsabilidad social, que, sin duda, deberá ejercer en beneficio de los otros y de la comunidad.

La obra de Davide Cali y la de los autores que la ilustran son, como se ha visto, de una enorme calidad por el mensaje subliminal que aparece en esta. Se trata de textos muy adecuados para el joven público lector que, con la ayuda de un mediador lector, puede acceder al sentido de estos. Este acceso se realiza a partir de una lectura atenta de un texto y de unas imágenes que han sido intencionadamente creados para él. De lo que se trata es de ofrecer ya en las primeras edades productos de lectura, como los álbumes presentados en este artículo, con la finalidad de trascender el sentido literario de estos y procurar una educación para la vida de calidad (Guerrero, Campos, Martos y Vázquez, 2011: 102). Los textos aquí presentados, sin duda alguna, contribuyen a la consecución de este doble objetivo.

\section{REFERENCIAS BIBLIOGRÁFICAS}

Azevedo, F., Balça, Â y Bastos, G. (2015). Web 2.0: literatura infantil/juvenil e comunidades interpretativas. Caderno seminal, 23, 40-57.

Azevedo, F., Selfa, M. y Balça, Â. (2016). La literatura juvenil publicada en Portugal: de las grandes tiradas comerciales a la interacción en la web 2.0. Revista Chilena de Literatura, 94, 103-118.

Ballester, J., Ibarra, N. y Haba, J. (2016). Diversidad y diferencia en la LIJ: una aproximación desde la censura contemporánea. En P. C. Cerrillo y C. Sánchez (Comps.), Prohibido leer: la censura en la literature infantile y juvenile contemporánea (pp. 365-372), Cuenca: Publicaciones de la Universidad de Castilla La Mancha.

Castells, M. La transición a la sociedad Red. Barcelona: Ariel, 2007.

Colomer, T. y Fernández de Gamboa Vázquez, K. (2014). La incorporación de la literatura infantil digital a la biblioteca de aula. Anuario de Literatura Infantil y Juvenil, 12, 19-36.

Dresang, E. T. (2008). Radical change revisited: dynamic digital age books for youth. Contemporary issues in technology and teacher education, 8/3, 294-304.

Duran, T. (2001). Àlbums i altres lectures. Anàlisi dels llibres per a infants. Barcelona: Rosa Sensat, 2001.

Faure, E., Herrera, F., Kaddoura, A., Lopes, H., Petrovski, A., Rahnema, M. y Champion, F. (1973). Aprender a ser. La educación del futuro. Madrid: Alianza Editorial. 
Guarisma, J. G. (2007). La educacion para la vida. Revista Iberoamericana de Educación, 44, 1-2.

Guerrero Rodríguez, M., Campos, M., Martos, A. E. y Vázquez, M. J. (2011). Cartografía de las prácticas lectoras. International Journal of Developmental and Educational Psychology, 1, 95-104.

Ibarra, N. y Ballester, J. (2010). La educación literaria e intercultural en la coonstrucción de la ciudadanía. Aula de Innovación Educativa, 197, 9.

Lage, J. J. (2006). Animar a leer desde la biblioteca. Madrid: CCS.

Lewis, D. (2001). Reading Contemporany Picture Books: Picturing Text. London-New York: Routledge-Farmer.

Martínez Ezquerro, A. (2016). El método de cooperación interpretativa como estrategia lectora. Álabe, 14. Recuperado de http://www.revistaalabe.com

Martínez Rodrigo, E. y González Fernández, A. M. (2010). La comunicación digital: nuevas formas de lectura y esritura, 63, 1-18.

Martos, E. y Campos Fernández-Figares, M. (Coords.) (2013). Diccionario de nuevas formas de lectura y escritura. Madrid: Santillana-Red Internacional de Universidades Lectoras. 